\title{
ON THE NATURE OF $\theta$ IN THE MEAN-VALUE THEOREM OF THE DIFFERENTIAL CALCULUS*
}

\section{BY GANESH PRASAD}

1. Introduction. If $f(x)$ is a single-valued function which is finite and continuous in an interval $(a, b)$, the ends being included, than the relation

$$
f(x+h)=f(x)+h f^{\prime}(x+\theta h), \quad 0<\theta<1,
$$

holds for every value of $x$ and $h$ for which the interval $(x, x+h)$ is in the interval $(a, b)$; provided that either $f^{\prime}(x)$ exists at every point inside the interval $(a, b)$ or a certain less restrictive condition $\dagger$ is satisfied. In recent years the nature of $\theta$ has been studied by a number of writers $\ddagger$ who start with the assumption that $f^{\prime \prime}(x)$ exists everywhere in the interval $(a, b)$. The two theorems, which it is the object of this paper to formulate and prove, are believed to be new and hold even if $f^{\prime \prime}(x)$ does not exist everywhere. For the sake of clarity and fixity of ideas, I consider $\theta$ only as a function of $h$, assuming $x$ to be a constant, say 0 , in the theorem $(\mathrm{M})$.

2. TheOrem I. If $\theta(h)$ is single-valued and continuous, it is not necessarily differentiable for every value of $h$.

Proof. Take $f(x)$ to be the indefinite integral of a monotone, increasing and continuous function which has a differential coefficient everywhere in the interval $(a, b)$, excepting the points

* Presented to the Society, February 25, 1928.

$\dagger$ The condition of W. H. Young and G. C. Young, Quarterly Journal of Mathematics, vol. 40 (1909), p. 1; Hobson's Theory of Functions of a Real Variable, vol. 1, 3d edition, 1927, p. 384; or the still less restrictive condition of A. N. Singh, Bulletin of the Calcutta Mathematical Society, vol. 19 (1928), p. 43.

$\ddagger$ R. Rothe (Mathematische Zeitschrift, vol. 9 (1921), p. 300; Tôhoku Mathematical Journal, vol. 29 (1928), p. 145); T. Hayashi (Science Reports of the Tôhoku Imperial University, (1), vol. 13 (1925), p. 385); O. Szász (Mathematische Zeitschrift, vol. 25 (1926), p. 116). 
of an everywhere dense set. Such a function is that given by T. Broden.* Denoting Broden's function by w, let

$$
f(x)=\int_{0}^{x} w(t) d t
$$

and let the everywhere dense set be denoted by $S$; also let $\xi$ stand for $h \theta$. Then it is easily seen that $\xi$ is a single-valued and continuous function of $h$, and that, corresponding to each value of $\xi$, there is a value of $h$ and only one value. Now (M) gives

$$
f(h)=h f^{\prime}(\xi)=h w(\xi),
$$

whatever $h$ may be.

Therefore, as $f^{\prime}(h)$ exists,

$$
\frac{d}{d h}\{h w(\xi)\}, \text { that is, } w(\xi)+h \frac{d w}{d h},
$$

must exist for every value of $h$. Thus, at any point $h=h^{\prime}$ which corresponds to a point $\xi=\xi^{\prime}$ of $S, d \xi / d h$ and, consequently, $d \theta / d h$ must be ron-existent; otherwise $w^{\prime}\left(\xi^{\prime}\right)$ will exist which is impossible.

Therefore it is proved that, for every value of $h$ corresponding to which $\xi$ is a point of $S, d \theta / d h$ is non-existent.

3. Theorem II. If $\theta(h)$ is single-valued, it is necessarily continuous for every value of $h$.

Proof. Assume, if possible, that $\bar{h}$ is a point of discontinuity of $\theta(h)$. Then, denoting the corresponding values of $\xi$ and $\theta$ by $\bar{\xi}$ and $\bar{\theta}$ respectively, we have by $(\mathrm{M})$

$$
f(\bar{h})=\bar{h} f^{\prime}(\bar{\xi}) .
$$

Now two possibilities arise: the discontinuity may be of the first kind or of the second kind.

(a) If the discontinuity is of the first kind, then there must be a sequence $\left\{h_{n}\right\}$, tending to $\bar{h}$, for which the corresponding sequence $\left\{\xi_{n}\right\}$ does not tend to $\bar{\xi}$ but to $\bar{\xi}^{\prime}$ different from $\bar{\xi}$. Thus

$$
f^{\prime}(\bar{\xi})=f^{\prime}\left(\bar{\xi}^{\prime}\right) .
$$

* Journal für Mathematik, vol. 118, p. 27; Hobson's Theory of Functions of a Real Variable, vol. 1, 1927, p. 389. 
So, for the same value of $h$, namely, $\bar{h}$, there are two values of $\theta$, namely, $\bar{\theta}$ and $\bar{\theta}^{\prime}$, which is absurd, since $\theta$ is single-valued.

(b) If the discontinuity is of the second kind, then there must be a sequence $\left\{h_{n}\right\}$, tending to $\bar{h}$, for which the corresponding sequence $\left\{\xi_{n}\right\}$ does not tend to any limit. Therefore two values $k_{1}$ and $k_{2}$ of $h$ can always be found as near as we please to $\bar{h}$ such that the corresponding values $\eta_{1}$ and $\eta_{2}$ of $\xi$ differ from each other by a quantity greater than a suitably prescribed positive quantity $\delta$. But, from $(\mathrm{M}), f(h) / h$ and, consequently, $f^{\prime}(\xi)$ are continuous functions of $h$ at $\bar{h}$. Therefore $\xi$ must be multiple-valued at $\vec{h}$, which is absurd, since $\theta$ is singlevalued.

The University of Calcutta

\section{A NUMERICAL FUNCTION APPLIED TO CYCLOTOMY}

BY EMMA T. LEHMER

A function $\phi_{2}(n)$ giving the number of pairs of consecutive integers each less than $n$ and prime to $n$, was considered first by Schemmel.* In applying this function to the enumeration of magic squares, D. N. Lehmer $\dagger$ has shown that if one replaces consecutive pairs by pairs of integers having a fixed difference $\lambda$ prime to $n=\prod_{i=1}^{t} p_{i}{ }^{\alpha_{i}}$, then the number of such pairs $(\bmod n)$ whose elements are both prime to $n$ is also given by

$$
\phi_{2}(n)=\prod_{i=1}^{t} p_{i}^{\alpha{ }^{-1}}\left(p_{i}-2\right) \text {. }
$$

As is the case for Euler's totient function $\phi(n)$, the function $\phi_{2}(n)$ obviously enjoys the multiplicative property $\phi_{2}(m) \phi_{2}(n)$ $=\phi_{2}(m n),(m, n)=1, \phi_{2}(1)=1$. In what follows we call an integer simple if it contains no square factor $>1$. For a simple number $n$ we have the following analog of Gauss' theorem:

$$
\sum_{\delta \mid n} \phi_{2}(\delta)=\phi(n)
$$

* Journal für Mathematik, vol. 70 (1869), pp. 191-2.

$\dagger$ Transactions of this Society, vol. 31 (1929), pp. 538-9. 